\section{Kompass Ophthalmologie}

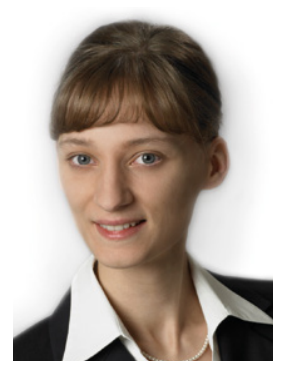

Bettina Hohberger

Koordination

«OphthalmoCampus»

\title{
Die Hornhaut des Auges
}

Liebe Leserinnen und Leser,

wie wichtig es für uns ist, dass jedes Organ und jedes Körperteil seine Funktion gut ausführen kann, wissen wir alle nur zu gut. Uns Augenärzten kommt hierbei eine so bedeutende Funktion zu - ist doch das Auge das für die meisten Menschen wichtigste Sinnesorgan. Es bedeutet Unabhängigkeit und Selbständigkeit. Ein schönes Beispiel hierfür kommt aus dem Bereich «Cornea»: Die Fuchs'sche Hornhautdystrophie ist gekennzeichnet durch eine progressive Visuseinschränkung, die es den Patienten über die Zeit hinweg erschwert, Ihren Alltag zu bewältigen. Was für ein Segen ist es für die Betroffenen, dass es die Möglichkeit gibt, dieser Erkrankung mit einer Hornhauttransplantation entgegen treten zu können. Wir geben Ihnen einen Einblick in die Hornhautbanken, die wunderbare Arbeit leisten, um das wertvolle Gewebe «Cornea» für unsere Patienten zur Verfügung zu stellen. Vieles in der Medizin ist nur dadurch möglich, dass sich Personen und Organisationen für die Gesundheit von anderen Menschen - auch ehrenamtlich - einsetzen. Ein weiterer Artikel stellt Ihnen eine neue Entwicklung in dem Bereich der Fuchs'schen Hornhautdystrophie vor, der es in Zukunft vielleicht ermöglichen könnte, alternativ zur Hornhauttransplantation, einen konservativen Weg gehen zu können.

Ich wünsche Ihnen viel Freude bei Lesen der aktuellen Ausgabe!

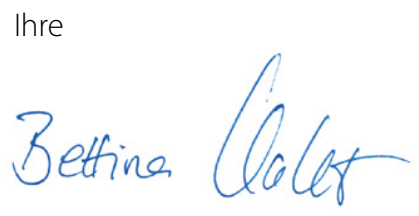

Bettina Hohberger 
Veranstaltungen und Kongresse

\section{Erster Lions Hornhautbank-Tag im Distrikt 111-Süd-Nord - erfreuliche Entwicklung der Lions Hornhautbank Heidelberg}

Eine erfolgreiche Patenschaft: Lions Clubs unterstützen seit sechs Jahren Hornhauttransplantationen an der Universitäts-Augenklinik Heidelberg. Jährlich erhalten rund 200 Patienten, die von Erblindung bedroht sind, eine neue Augenhornhaut. Lions Clubs finanzieren Projekte und Ausrüstung für eine bessere Versorgung mit Hornhauttransplantaten in Nordbaden.

\section{Die Fakten}

Die Hornhaut ist gewissermaßen die Windschutzscheibe des Auges. Sie überdeckt wie ein gewölbtes Uhrglas Pupille und Iris. Gemeinsam mit der Augenlinse bündelt sie das einfallende Licht und hat einen erheblichen Anteil am scharfen Sehen. Darüber hinaus schützt sie das Auge vor Fremdkörpern und Krankheitserregern. In Folge von Verletzungen, Infektionen, Entzündungen oder angeborenen Erkrankungen kann sie sich teilweise oder vollständig eintrüben. Betroffene können dann trotz eines ansonsten gesunden Auges sogar erblinden. Diese Form der Erblindung lässt sich durch die Transplantation der Augenhornhaut eines Verstorbenen verhindern bzw. rückgängig machen. Die Hornhautübertragung gilt heute in Europa als Routineeingriff in der Augenchirurgie.

Anders als bei Organen können die Spenderhornhäute durch eine anspruchsvolle Aufbereitung und in speziellen Brutschränken noch mehrere Wochen funktionsfähig erhalten werden. Nach eingehender Untersuchung unter anderem auf Schäden, Lebensfä- higkeit und übertragbare Krankheiten lagern sie bis zur Transplantation in einer speziellen Nährlösung bei einer Temperatur von 34,4 Grad Celsius, die der im Auge entspricht.

Rund 200 Patientinnen und Patienten, die akut von Erblindung bedroht sind, erhalten jährlich an der Universitäts-Augenklinik Heidelberg eine Hornhauttransplantation, Tendenz steigend. Voraussetzung für diese positive Entwicklung ist die gut aufgestellte Gewebebank für Augenhornhäute an der Klinik, die nun schon seit sechs Jahren von den 58 Lions Clubs des Distrikts 111-SN gefördert wird. In ihr werden die Gewebespenden Verstorbener aufbereitet und in speziellen Brutschränken gelagert.

\section{Etwas Geschichte}

Das Engagement der Lions Clubs im Kampf gegen vermeidbare Blindheit habe eine beinahe 100-jährige Tradition, insbesondere die Zusammenarbeit mit Hornhautbänken weltweit, wie DistriktGovernor Werner H. Schwenk anlässlich seiner Begrüßung der Gäste an der Veranstaltung ausführte: Bereits die erste, in New York gegründete Hornhautbank wurde seit 1952 von den Lions unterstützt. Inzwischen sind es weltweit 45, stolze 7 in Deutschland. «In dieser Tradition haben wir im März 2015 die Patenschaft für <unsere> Hornhautbank in Heidelberg übernommen», sagt er. «Veränderungen der Hornhaut des Auges zählen zu den weltweit häufigsten Ursachen für Blindheit bzw. schwere Sehbehinderung. Durch die

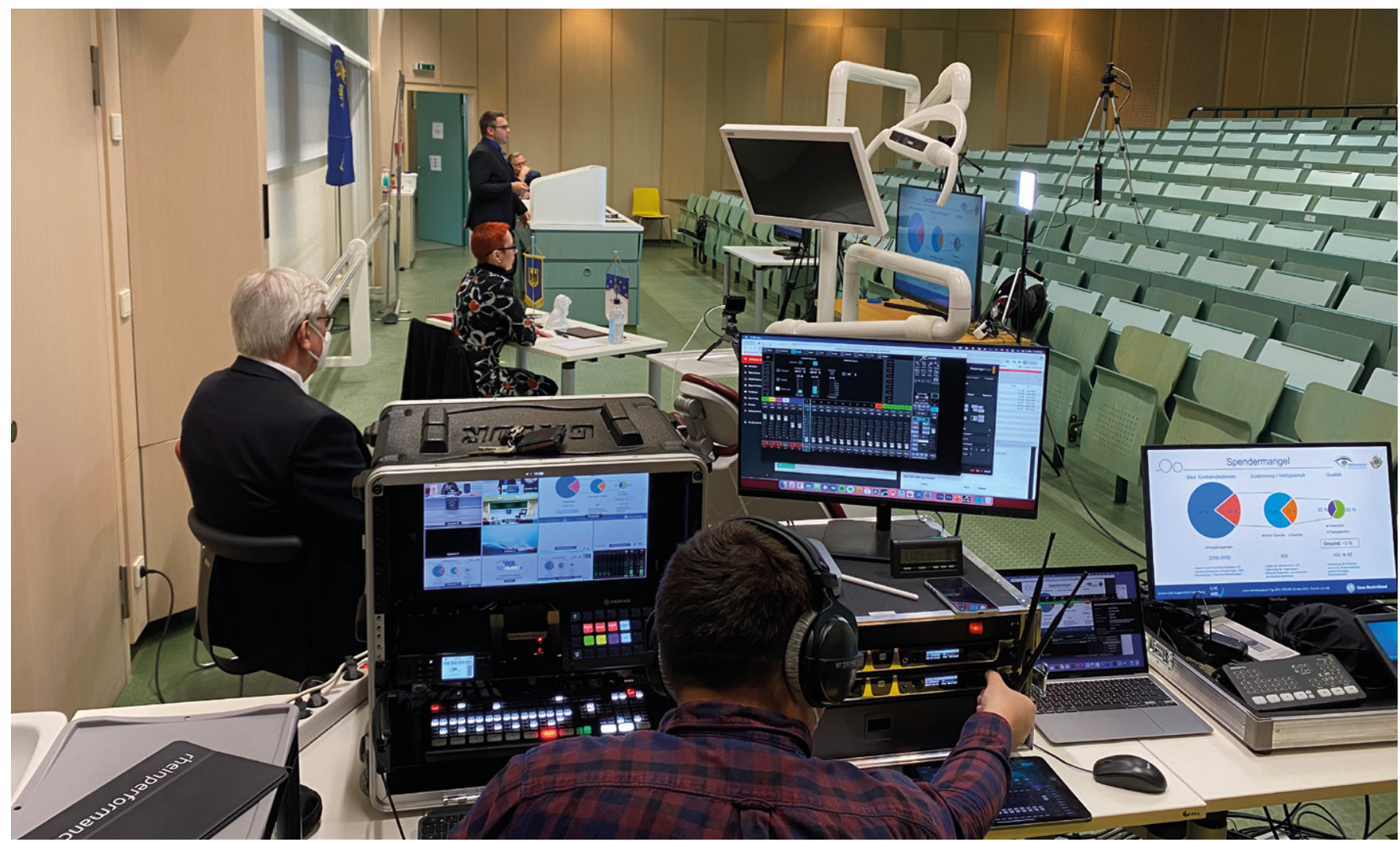

Abb. 1. Das technische Set-Up der Veranstaltung. 


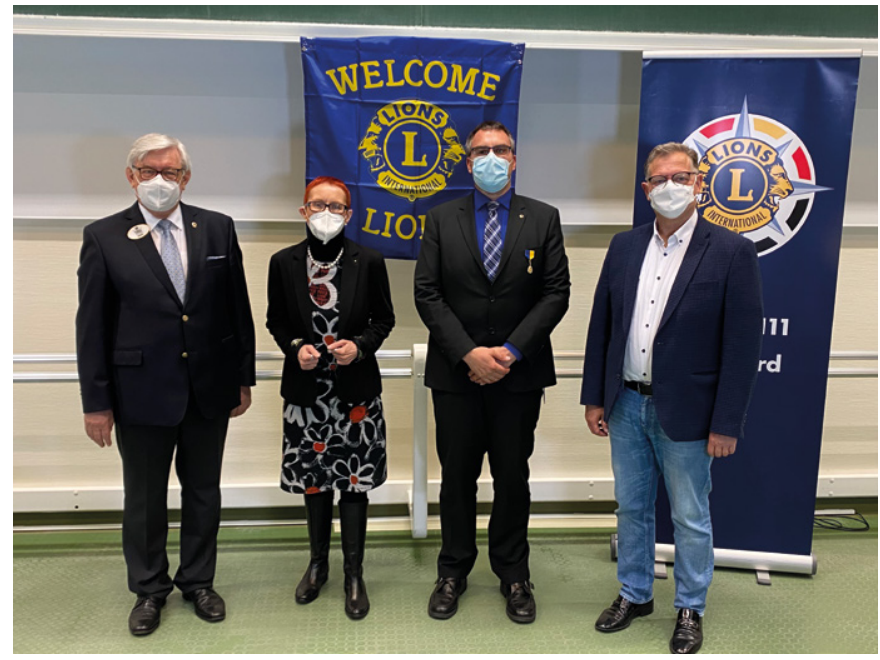

Abb. 2. Das Studio-Team vor Ort - Corona-konform! (vInr: Werner Schwenk, Dr Sibylle Scholtz, Dr. Patrick Merz, Prof. Dr. Gerd Auffarth)

Förderung von Hornhautbanken tragen wir unseren Teil dazu bei, möglichst vielen Menschen mit Erkrankungen oder Verletzungen der Augenhornhaut ein Leben in Blindheit zu ersparen.»

\section{Meilensteine}

Vor dem Hintergrund der ersten Pandemiewelle fiel die ursprünglich für 2020 geplante Feier zum 5-jährigen Jubiläum dieser «Patenschaft» leider aus. Um die Leistungen der Lions Hornhautbank adäquat zu würdigen, lud Distrikt-Governor Werner H. Schwenk für den 1. Mai 2021 zum ersten Lions Hornhautbank-Tag 2021 in die Lions Hornhautbank an der Universitäts-Augenklinik Heidelberg ein, um das 6-jährige Bestehen dieser Lions Institution als HybridVeranstaltung zu feiern und auch allen Interessierten zu präsentieren (Abb. 1). Gewissermaßen nachträglich zog das Team der «Lions Hornhautbank Heidelberg» am 1. Mai 2021 eine höchst positive Bilanz: Professor Dr. Gerd U. Auffarth, Ärztlicher Direktor der Universitäts-Augenklinik und medizinischer Leiter der Hornhautbank, Dr. Patrik Merz, wissenschaftlicher Leiter der Lions-Hornhautbank, sowie Werner H. Schwenk, Distrikt-Governor des Distrikts 111-SüdNord, und Dr. Sibylle Scholtz, Kabinettsbeauftragte Lions Hornhautbank und Sight First, warfen bei der virtuellen Feierstunde, die von der Düsseldorfer Congress-Organisation Gerling höchst professionell als Hybrid-Veranstaltung durchgeführt wurde, sowohl einen Rückblick auf die bisherige fruchtbare Partnerschaft als auch erste Blicke auf zukünftige Projekte.

Am 27. März 2015 wurde die Hornhautbank Heidelberg im Amtsjahr von Distrikt-Governor Uwe Metzinger als fünfte Lions Hornhautbank in Deutschland und weltweit als 54. Lions Hornhautbank gegründet. Alle Lions Clubs der Region Nordbaden übernahmen damit auch für «ihre» Hornhautbank die Patenschaft und die Verantwortung, sie unterstützen seither zahlreiche Projekte und Anschaffungen dieser Einrichtung.

Der nächste Meilenstein in der Geschichte der Lions Hornhautbank in Heidelberg konnte am 20. November 2016, im Lions Jubiläumsjahr 2016/2017 anlässlich des 100-jährigen Bestehens von Lions
Clubs International, gefeiert werden: Dieses «Leuchtturmprojekt» wurde vom seinerzeitigen Distrikt-Governor Dr. Michael Pap in den Stand eines «Centennial Legacy Projekts des Distrikts 111-SüdNord» erhoben. Die in der Klinik angebrachte Gedenktafel zeugt heute von dieser außergewöhnlichen Ehrung der Lions und der Würdigung der Arbeit dieser Institution. Auch der Besuch des internationalen Präsidenten der Lions Organisation, Dr. Naresh Aggarval, am 3. Oktober 2017 zeigt deutlich die Wertschätzung, die der Lions Hornhautbank Heidelberg auch international innerhalb der Lions Organisation entgegengebracht wird.

«In den sechs Jahren der Lion-Patenschaft haben wir die Anzahl der Transplantationen und der aufbereiteten Gewebespenden fast verdoppelt. Auch unsere Forschung auf dem Gebiet der Hornhauttransplantation macht große Fortschritte», freut sich Prof. Auffarth, der die Lions Hornhautbank leitet. «Diese Erfolge sind nicht zuletzt der kontinuierlichen Unterstützung der Lions zu verdanken, die in dieser Zeit nicht nur einen Kleinwagen für die nötige Mobilität bei der Spendenakquise, ein Spezialmikroskop sowie einen SpezialBrutschrank spendeten, sondern auch die Fort- und Weiterbildung des Teams auf Kursen und Kongressen regelmäßig mittragen.»

\section{Der Festakt}

Aus dem «Übertragungs-Studio», zu dem der große Hörsaal der Uni-Augenklinik umfunktioniert worden war (Abb. 1), präsentierten DG Werner H. Schwenk, KSF Dr. Sibylle Scholtz, der medizinische Leiter der Lions Hornhautbank Prof. Dr. Auffarth und Dr. Patrick Merz als wissenschaftlicher Leiter der Hornhautbank den über einhundert Teilnehmern der Online-Veranstaltung einen bunten Strauß an Informationen (Abb. 2). Dr. Merz stellte die tägliche Arbeit in der Hornhautbank vor, Prof. Dr. Auffarth referierte an Hand von anschaulichen und beeindruckenden Foto- und Filmaufnahmen über moderne Transplantationstechniken der Hornhaut. Ein virtueller Rundgang durch die Hornhautbank mittels eines Videos, das Prof. Auffarth und Dr. Merz speziell für diese Veranstaltung erstellt hatten, rundete das sehr beeindruckende Bild weiter ab.

Auch Ehrungen durften bei dieser besonderen Veranstaltung nicht fehlen: DG Schwenk ehrte die für die Lions Hornhautbank im DistriktKabinett zuständige KSF Dr. Sibylle Scholtz für ihre über 6-jährige Arbeit an und mit der Hornhautbank mit einem Progressive Melvin Jones Fellow Award. Dr. Patrick Merz, Mitglied im LC MannheimRosengarten, wurde mit einem Distrikt-Governor Activity Award und Prof. Dr. Gerd U. Auffarth mit einer Medal of Merit ausgezeichnet. Eine Aufzeichnung der Veranstaltung, die höchst professionell von der Congress-Organisation Gerling GmbH Düsseldorf vorbereitet und durchgeführt wurde, kann über diesen Link aufgerufen werden: www.lions.de/web/111sn.

\section{Eine fruchtbare Zusammenarbeit}

Bereits 1999 wurde Universitätsklinikum Heidelberg eine Hornhautbank eingerichtet. Vor der Umwandlung in eine Lions Hornhautbank 2015 mussten die in der Universitäts-Augenklinik benötigten Hornhäute größtenteils von anderen Gewebebanken zugekauft werden. «Das hat sich dank der Unterstützung der Lions, aber auch durch die Kooperation mit der Deutschen Gesellschaft für 
Gewebetransplantation (DGFG), inzwischen geändert», sagt Prof. Auffarth. Durch die kontinuierliche Schulung des Teams können die Gewebeentnahmen in den Kliniken der Region selbst durchgeführt und so mehr Hornhautspenden akquiriert werden. Die moderne technische Ausstattung ermöglicht es, aktuelle Entwicklungen der Transplantationstechnik umzusetzen und die Operationszahlen kontinuierlich zu steigern. «Damit hat sich in den letzten sechs Jahren nicht nur die Versorgung verbessert, sondern auch die Wartezeiten der einzelnen Patienten von maximal zwei Jahren auf durchschnittlich rund ein bis zwei Monate verkürzt», so der Augenchirurg. In Heidelberg stehen derzeit um die 50 Patienten auf der Warteliste für eine Hornhauttransplantation, deutschlandweit sind es rund 7000 bei gleichzeitig etwa 9000 Transplantationen pro Jahr. Ähnlich wie auch bei Organtransplantationen müssen die Gewebemerkmale der Hornhaut möglichst genau zu denen des Empfängers passen. Die Zusammenarbeit mit Deutschlands größtem Netzwerk für Gewebemedizin, der DGFG, erleichtert seit 2018 die Vermittlung passender Hornhäute. «lst eine passende Hornhaut bei uns nicht auf Lager, dann vermittelt die DGFG ein Gewebetrans- plantat aus einer der anderen Hornhautbanken des Netzwerks», sagt Dr. Patrick Merz, wissenschaftlicher Leiter und Qualitätsmanagementbeauftragter der Lions Hornhautbank Heidelberg. Ebenso stellt die Heidelberger Gewebebank aufbereitete Hornhäute für Patienten der kooperierenden Kliniken zur Verfügung.

In den gut 2 Stunden Hybrid-Meeting bot der Hornhautbank-Tag der Lions Hornhaubank Heidelberg einen sehr detaillierten Einblick in die aufwändige Arbeitsweise einer Hornhautbank und vermittelte auf verständliche Weise wissenschaftliche Hintergründe der verschiedenen Techniken einer Hornhauttransplantation. Man darf auf die Feier zum 10-jährigen Jubiläum gespannt sein!

\section{Autoren:}

Dr. Sibylle Scholtz, Kabinettsmitglied für SightFirst und Lions Hornhautbank Uni Augenklinik Heidelberg Lions Distrikt 111-Süd-Nord Werner Schwenk, Distrik Governor, Lions Distrikt 111-Süd Nord

(c) alle Fotos: Sven Gerling, Düsseldorf

\section{Ophthalmologie kompakt}

\section{«Fuchs, du hast die Sicht gestohlen ...»}

«...Gib sie wieder her!» So die Hoffnung vieler Patienten, die von der Fuchs'schen Hornhautdystrophie (engl. «Fuchs Endothelial Corneal Dystrophy» - FECD) betroffen sind. Doch wie könnte dieser Wunsch in Erfüllung gehen? Ist das ohne einen invasiven operativen Eingriff überhaupt möglich?

Um diese Fragen zu klären, vorab ein paar Worte zur Erkrankung: Die von Ernst Fuchs im Jahre 1902 erstmals beschriebene Veränderung der innersten Schicht der Kornea - dem Hornhautendothel und seiner Basalmembran, der «Descemet-Membran» (DM) - ist durch den Untergang ebenjener Endothelzellen gekennzeichnet. Somit fällt in immer größer werdenden Arealen der Hornhaut die Funktion der Endothelzellen weg. Diese sorgen hauptsächlich für den Erhalt des osmotischen Gleichgewichts zwischen Hornhautstroma und Kammerwasser und produzieren außerdem die extrazelluläre Matrix (EZM), aus der die DM besteht. Ohne die Pumpfunktion der Zellen entstehen korneale Ödeme, die zu einer Visusverschlechterung führen. Zudem sorgt die pathologische Überproduktion von EZM durch die verbliebenen Endothelzellen für eine Verdickung der Hornhaut und warzenartige Ausstülpungen der DM - den sog. Guttae. Es beginnt zumeist mit einer zentralen «Cornea guttata», die sich im weiteren Verlauf der Erkrankung peripher ausbreitet. Die Symptome umfassen folglich verschwommene Sicht, schlechte Nachtsicht und Schmerzen beim Blinzeln, die unbehandelt bis zu einer Erblindung führen können.

Nicht weiter verwunderlich also, dass die FECD eine der häufigsten Ursachen für eine Hornhauttransplantation ist. State-of-theart ist hierbei die Vorgehensweise einer «Descemet Membrane Endothelial Keratoplasty» (DMEK), bei der in einem minimalinvasiven Verfahren die erkrankte Endothelschicht samt DM abgezogen und durch ein gesundes Transplantat ersetzt wird. Mit die- ser Methode können gute langfristige Resultate erzielt werden, jedoch kann ein nochmaliges Zugrundegehen der Endothelzellen nach Jahren nicht gänzlich ausgeschlossen werden, da die Hauptursache für die Entstehung der FECD noch nicht bekannt ist. Wir wissen jedoch bereits, dass UV-Belastung, Alter, Geschlecht, Diabetes, Rauchen und teilweise auch eine genetische Veranlagung Risikofaktoren für die FECD sind. Ein operativer Eingriff birgt natürlich immer auch gewisse Risiken, die man beispielsweise bei einer schwach ausgeprägten Form der Erkrankung nicht unbedingt eingehen möchte. Ein weiterer limitierender Faktor dieser Therapie ist zudem das Vorhandensein von genügend Spenderhornhäuten [1].

Aber nun scheint die Erfüllung des Wunsches nach einer konservativen Behandlung, durch die die pathologische Veränderung der Endothelzellen verzögert, aufgehalten oder sogar geheilt werden könnte, jetzt endlich zum Greifen nahe zu sein!

Dazu macht man sich ein Medikament zunutze, das schon aus anderen Bereichen der Augenheilkunde (z.B. Glaukom) bekannt ist: Ripasudil (Handelsname Glanatec ${ }^{\circledR}$ ), ein Inhibitor der Rho-Kinasen (ROCK). Im Tierversuch an Primaten konnte die positive Wirkung von ROCK-Inhibitoren bereits gezeigt werden: Die zentral gelegenen Endothelzellen wurden bei den Tieren durch transkorneale Vereisung zerstört und die Tiere anschließend mit einem ROCKInhibitor in Form von Augentropfen behandelt. Dieses Vorgehen förderte die Wundheilung durch die verbliebenen, randständigen Zellen, somit also die Proliferation und Migration in die endothelzellfreien Areale [2]. An der Augenklinik der Universität Erlangen wird aktuell im Rahmen einer prospektiven, monozentrischen, randomisierten Phase-II-Studie die Behandlung der FECD mit Glanatec ${ }^{\circledR}$ klinisch erforscht [3]. Ergänzend dazu wird in den mole-
144

Kompass Ophthalmol 2021;7:141-145 DOI: $10.1159 / 000518688$ 
kularbiologischen Laboren der Augenklinik unter der Leitung von Frau Prof. SchlötzerSchrehardt die Wirkung des ROCK-Inhibitors grundlagenwissenschaftlich erforscht [4]. Die Ergebnisse sind noch nicht vollständig - zeigen aber schon jetzt einen klaren Trend, der für einen vermehrten Einsatz von ROCK-Inhibitoren in der Therapie der FECD spricht. Somit könnte für einige Patienten in Zukunft der Jäger mit dem Schießgewehr alias Operateur mit dem Skalpell nicht mehr zwingend notwendig sein. Es bleibt spannend!

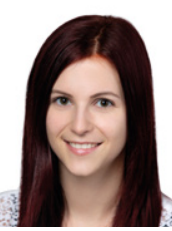

Autorin: Maria Strunz

\section{Literatur}

1 Nanda GG, Alone DP: REVIEW: Current understanding of the pathogenesis of Fuchs' endothelial corneal dystrophy. Mol Vis. 2019;25:295-310.

2 Okumura N, Koizumi N, Kay EP, et al.: The ROCK inhibitor eye drop accelerates corneal endothelium wound healing. Invest Ophthalmol Vis Sci. 2013;54(4):2493-2502.

3 Universitätsklinikum Erlangen: Augentropfen statt Transplantation als Option bei Fuchs'scher Endotheldystrophie? www.augenklinik.uk-erlangen.de/ aerzte-und-zuweiser/informationen/augentropfenstatt-transplantation

4 Schlötzer-Schrehardt U, Zenkel M, Strunz M, et al. Potential functional restoration of corneal endothelial cells in fuchs endothelial corneal dystrophy by ROCK inhibitor (Ripasudil). Am J Ophthalmol. 2021;224:185-199.

\section{Steckbrief: Fuchs Endothelial Corneal Dystrophy (FECD)}

\begin{tabular}{ll}
\hline Symptome & $\begin{array}{l}\text { Verschwommene Sicht, schlechte Nachtsicht, Schmerzen } \\
\text { beim Blinzeln, Erblinden (unbehandelt) }\end{array}$ \\
\hline Pathophysiologie & $\begin{array}{l}\text { Zugrundegehen des Hornhautendothels; dadurch korneale } \\
\text { Ödeme, Verdickung der Descemet-Membran (DM), warzen- } \\
\text { artige Ausstülpungen der DM (Guttae) }\end{array}$ \\
\hline Ursachen & $\begin{array}{l}\text { Hauptursache unbekannt; genetische Faktoren, UV- } \\
\text { Belastung, Alter, Geschlecht, Diabetes, Rauchen }\end{array}$
\end{tabular}

Relativ selten, aber häufigste Ursache für Hornhauttransplan-

\begin{tabular}{ll} 
Häufigkeit & $\begin{array}{l}\text { tationen in den USA; häufigste Erkrankung des kornealen En- } \\
\text { dothels }\end{array}$ \\
\hline Geschlecht & Frauen sind 3-4 Mal häufiger betroffen \\
\hline Alter & $>40$ Jahre \\
\hline
\end{tabular}

- Alter 3-40 Jahre

- Seltener

Frühe Form - Schwereres Krankheitsbild

- Meist erblich (autosomal dominant)

- Tiefere und unzusammenhängende Guttae

- Starke Verdickung der DM

\begin{tabular}{|c|c|c|}
\hline Arten & Späte Form & $\begin{array}{l}\text { - Alter }>40 \text { Jahre } \\
\text { - häufiger } \\
\text { - leichteres Krankheitsbild } \\
\text { - meist nicht erblich } \\
\text { - flache und zusammenhängende Guttae } \\
\text { - weniger starke Verdickung der DM } \\
\text { - zusätzliche «posterior banded layer» in der Kornea möglich }\end{array}$ \\
\hline Aktuel & erapie & et Membrane Endothelial Keratoplasty (DMEK) \\
\hline
\end{tabular}

